\title{
miR-21 modulates the effect of EZH2 on the biological behavior of human lung cancer stem cells in vitro
}

\author{
Hui Xia ${ }^{1,2,3}$, Wen Zhang ${ }^{1}$, Baoshi Zhang ${ }^{1}$, Yingnan Zhao ${ }^{1}$, Yunlong Zhao ${ }^{1}$, Shaojun \\ Li $^{1}$ and Yang Liu ${ }^{2}$ \\ ${ }^{1}$ Department of Thoracic-Cardio Surgery, First Affiliated Hospital of PLA General Hospital, Beijing, China \\ ${ }^{2}$ Department of Thoracic Surgery, PLA General Hospital, Beijing, China \\ ${ }^{3}$ Medical School of PLA, Beijing, China \\ Correspondence to: Hui Xia, email: 304xiahui@sina.com
}

Yang Liv, email: China liuyangplats@126.com

Keywords: miR-21, EZH2, lung cancer stem cells, radiotherapy, chemotherapy

Received: November 10,2016 Accepted: June 19, $2017 \quad$ Published: August 07, 2017

Copyright: Xia et al. This is an open-access article distributed under the terms of the Creative Commons Attribution License 3.0 (CC BY 3.0), which permits unrestricted use, distribution, and reproduction in any medium, provided the original author and source are credited.

\section{ABSTRACT}

Non-small-cell lung cancer has a high mortality rate and poor prognosis. Therefore, novel therapeutic approaches are urgently needed to enhance patient survival rates. In this study, we investigated the effects of miR-21 and EZH2 on the biological behavior of human lung cancer stem cells in vitro. We found increased expression of EZH 2 and miR-21 in LCSCs, and miR-21 overexpression increased EZH2 levels in LCSCs. In addition, EZH2 and miR-21 knockdown increased the sensitivity of LCSCs to chemo- and radiation therapy, and exogenous EZH2 expression rescued the effects of anti-miR-21. Cell proliferation was reduced by $39.2 \%$ and $69.7 \%$ in the presence of radio- or chemotherapy combined with anti-miR-21 transfection, respectively. The downstream molecules included Cdc2, cyclin B1, and Bcl-2, which are involved in the regulation of cell cycle and apoptosis and which could themselves be reduced or enhanced by changes in miR-21 and EZH2 levels in LCSCs. This study demonstrates the direct relationship between miR-21 and EZH2 which was increased by $43 \%$ after the application of the miR-21 mimic. Above data indicates that these two molecules can influence the biological behavior of LCSCs by altering their corresponding targets. Our findings support the potential roles of miR-21 and EZH2 in improving the therapeutic efficacy of clinical lung cancer treatments.

\section{INTRODUCTION}

Lung cancer, the leading cause of cancer-related mortality worldwide, has a high mortality rate and poor prognosis-particularly non-small-cell lung cancer (NSCLC), which accounts for approximately $80 \%$ of all lung cancers and has a 5-year overall survival rate of $<$ $15 \%[1,2]$. In addition, approximately $40 \%$ of all NSCLC patients present with unresectable stage III disease or inoperable disease $[3,4]$.

Despite recent advances and developments in therapeutic strategies, including surgery, chemotherapy, and radiotherapy, poor survival remains an issue due to the high systemic toxicity and drug resistance that limit successful outcomes in most cases [5, 6]. Therefore, the development of novel approaches for the diagnosis, treatment, and prevention of NSCLC, including targeted gene therapy as an adjuvant modality or as a radiosensitizer, is urgently needed to enhance patient survival.

Cancer stem cells, which exhibit stem cell-like selfrenewal capabilities, are involved in the initiation and development of cancers such as breast [7], pancreatic [8], brain [9] and lung cancers [10]. Recent studies [11, 12] and our previous report [13] supported the role of side population (SP) cells, which can be isolated from human lung cancer cell lines and exhibit significant tumorinitiating activity and characteristic proteins, such as 
CD133, ABCG2 and multi-drug resistance transporters. Lung cancer stem cells (LCSCs) represent a novel target for clinical cancer therapy, but the underlying mechanisms for LCSC tumorigenesis remain unknown and is an urgent area of study.

Recently, significant advances in our biological and molecular understanding of cancer have facilitated the development of agents targeted to specific molecules in the treatment of NSCLC [14, 15]. Novel anti-lung cancer agents that target proteins such as EGFR or Enhancer of zeste homolog 2 (EZH2) combined with chemotherapy or radiotherapy have been reported [16, 17]. EZH2, a catalytically active component of the PRC2 complex, is one of the targets currently being evaluated for the treatment of lung cancer. Various studies have identified that abnormal expression of EZH2, a potential marker for distinguishing aggressive from indolent or benign cancers, contributes to the tumorigenesis of several malignancies, including melanoma, prostate, breast, bladder, and endometrial cancers, and results in proliferative advantages for eukaryotic cells by affecting the key pathways that control cellular growth arrest and differentiation $[18,19]$. As a transcriptional repressor, EZH2 controls cellular growth and proliferation by promoting S-phase entry and the $\mathrm{G}_{2} / \mathrm{M}$ transition $[20,21]$. EZH2 also promotes the repression of specific genes, a process that also involves histone deacetylation by histone deacetylase-1 (HDAC-1), which interacts with EZH2 via its PRC2-binding partner EED [22, 23].

microRNAs (miRNAs) are a class of short noncoding RNAs that have been demonstrated to regulate the expression of genes governing tumorigenic processes by targeting mRNAs for degradation or translational inhibition. miRNAs play key roles in lung cancer development, including cellular differentiation, apoptosis, invasion and the cell cycle [24-26]. miR21 is overexpressed in several human malignancies, including NSCLC. miR-21 expression in lung cancer can be considered a biomarker for poor prognosis, chemotherapeutic response and radioresistance [27-29]. miR-21 has been demonstrated to play a important role in the radioresistance of cancers, including glioblastoma, breast cancer, rectal cancer. The inhibition of miR21 expression sensitizes cancer cells to topotecan and gemcitabine [30-31]. miR-21 can modulate the histone deacetylase (HDAC) expression and Akt/Gsk3 $\beta$ pathway [32]. Our recent study also demonstrated that the down regulation of miRNA-21 sensitizes radioresistant NSCLC A549 cells to IR by inhibiting the PI3K/Akt signaling pathway [33]. In addition, effect of EZH2 mediated epigenetic gene silencing is dependent on HDAC activity [34-35]. And our data also reported that EZH2 regulate cell cycle through its SET-domain regulated H3K27me3 activity via p53/p21 downstream pathway [36].

Few studies have reported on the function of miRNAs, particularly miR-21, in LCSCs. Thus, in this study, we tested the hypothesis that down regulation of miR-21 and EZH2 expression level via anti-miR-21 or EZH2 shRNA reduce LCSC growth, thereby altering lung cancer development and progression. The underlying mechanism and the related pathway involving miR-21 and EZH2, which are important biomarkers and target molecules in the clinical treatment for lung cancer, were explored. Our results provide direct evidence for the application of miR-21 or EZH2 knockdown in future clinical treatment strategies for NSCLC patients.

\section{RESULTS}

\section{EZH2 expression in lung cancer stem cells}

To detect EZH2 in LCSCs, we performed realtime quantitative RT-PCR and western blotting analyses. Both analyses revealed high levels of EZH2 in LCSCs (Figure 1, Supplementary Figure 1). These results were consistent with previous reports $[37,38]$, which previously indicated a relationship between EZH2 expression and lung cancer development.

Two independent shRNAs were used to knockdown EZH2 to assess its functional significance in LCSC. Both EZH2 shRNAs significantly decreased EZH2 expression at the protein and mRNA levels. In addition, we observed toxicity in LCSCs after the transfection of these two EZH shRNAs. The GFP- positive populations of live cells were normalized to that of the control groups (negative shRNA) first and to the day-2 fraction (Figure 1). Each experiment was performed independently at least 3 times.

We used the specific pharmacological inhibitor GSK343 to down regulate expression level of H3K27me3 and EZH2 activity. Next, cell viability was evaluated after 4 days of incubation with GSK343. We observed that the effect of GSK343 on cell growth was expressed in dose-dependent manner, and $1 \mu \mathrm{M}$ GSK343 reduced cell viability by approximately 50\% (Figure 1). These data indicate that the pharmacological inhibitor can mimick EZH2 knock down which affects LCSC survival and represents a potential therapeutic modality for lung cancer patients.

\section{Effect of miR-21 on EZH2 expression}

We observed high expression levels of miR-21 in LCSC by real-time quantitative RT-PCR, as well as its down regulation after transfection with anti-miR-21 compared with controls (Figure 2). Our western blotting results suggested that EZH2 expression was down regulated by the application of anti-miR-21, as the levels decreased by $58 \%$ compared with controls. Transfection of anti-miR-21 can induce toxicity in LCSCs (Figure 2). To determine whether the effects of miR-21 down regulation on cell proliferation are related to the expression of 
$\mathrm{EZH} 2$, a rescue experiment was performed to observe the effects of EZH2 up regulation. Indeed, exogenous EZH2 expression rescued LCSCs from anti-miR-21-induced toxicity (Figure 2). Furthermore, miR-21 over expression through transfection with a miR-21 mimic (OE miR21) also increased EZH2 expression. Compared with controls, EZH2 expression was increased by $43 \%$ after the application of the miR-21 mimic (Figure 2). Based on theses results, miR-21 appears to enhance EZH2 expression in LCSCs.

\section{Colony-formation assays}

We performed colony-formation assays to assess the effects of EZH2 knockdown with shRNA or antimiR-21 treatment. Both EZH2 and miR-21 knockdown significantly reduced colony formation. GSK343 elicited similar effects. In addition, we performed an EZH2 cDNA mutant experiment to rescue the effect of anti-miR-21 treatment. Our results indicated that the colony-formation capacity that was reduced by anti-miR- 21 could be rescued by the over expression of exogenous EZH2 (Figure 3).

\section{Migration and invasion assays}

We evaluated the effects of miR-21 and EZH2 on cell migration and invasion by down or up regulating miR-21 and EZH2 expression. Compared with controls, the suppression of either miR-21 or EZH2 resulted in significantly decreased well migration and invasion activity. Similarly, exogenous EZH2 over expression rescued the effects of anti-miR-21 in LCSCs (Figure 3). Thus, these data demonstrate that both miR-21 and EZH2 are important for cell migration and invasion.

\section{Effects on cell proliferation}

In the following experiments, we tested the hypothesis that changes in miR-21 or EZH2 expression influence the effects of radio- or chemotherapy on LCSC proliferation. After treatment with radiotherapy alone, the number of LCSCs was reduced by $28.5 \%$, and a significant effect was observed in the presence of EZH2 shRNA, where it was reduced by at least $51.3 \%$ (Figure 4 ). In addition, our results indicate that the reduction of miR-21
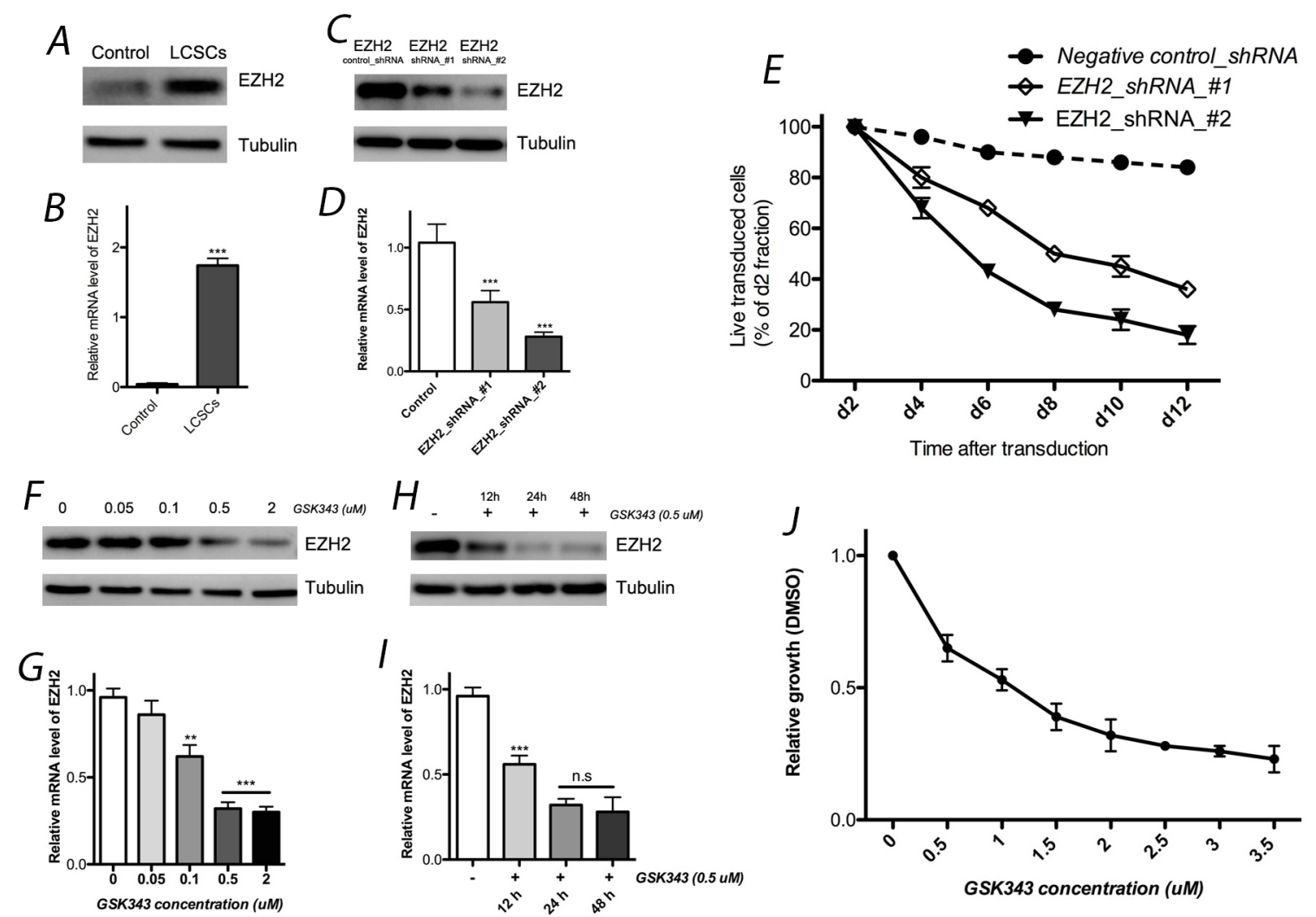

Figure 1: EZH2 expression in LCSCs. EZH2 expression in LCSCs by western blotting (A) and real-time quantitative RT-PCR (B) analyses. Both EZH2 shRNAs significantly decreased EZH2 expression in LCSCs at the protein (C) and mRNA (D) levels; (E) effects of EZH2_shRNA on cell growth of LCSCs; EZH2 protein $(\mathbf{F})$ and mRNA (G) expression was affected by different concentration of GSK343; effect of GSK343 with different incubation time were also observed by western blotting (H) and real-time quantitative RT-PCR (I) analyses; (J) cell viability was evaluated after 4 days of incubation with GSK343. Each experiment was performed independently at least 3 times. Each experiment was performed in triplicate, ${ }^{* * *} \mathrm{P}<0.001,{ }^{* *} \mathrm{P}<0.01,{ }^{*} \mathrm{P}<0.05$. 
by anti-miR-21 transfection also resulted in additive antiproliferative effects on cells when combined with radiation therapy, where the number of LCSCs was reduced by $39.2 \%$ and $26.3 \%$ in the presence or absence, respectively, of anti-miR-21 transfection.

In cisplatin-treated cells, the number of cells was reduced by at least $29.7 \%$, and an additive effect was observed in the presence of EZH2 shRNA, where cell numbers were reduced by $55.1 \%$. When combined with anti-miR-21 transfection, the number of cells was further reduced by $69.7 \%$ after chemotherapy and $31.2 \%$ for the group that received cisplatin alone. Thus, our data demonstrate that EZH2 shRNA or anti-miR-21 can elicit additive anti-proliferative effects when combined with chemo- or radiotherapy on LCSCs. Interestingly, the rescue effect of anti-miR-21 was also observed after the ectopic expression of EZH2 (Figure 4).
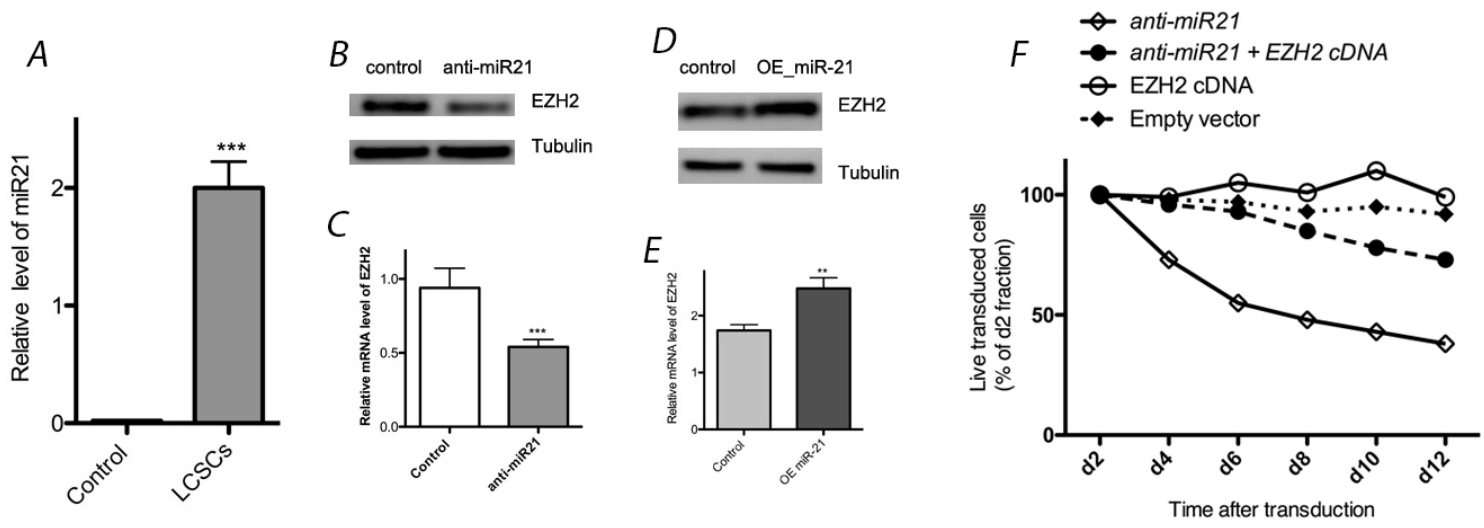

Figure 2: Effect of miR-21 on EZH2 expression in LCSCs. (A) Relative expression of miR-21 was detected in LCSC by real-time quantitative RT-PCR; (B) \& (C) EZH2 expression was reduced by transfection with anti-miR-21 in LCSC; (D) \& (E) EZH2 expression was enhanced by transfection with OE miR-21 in LCSC; $(\mathbf{F})$ transfection of anti-miR-21 can induce toxicity in LCSCs, and exogenous EZH2 expression rescued LCSCs from anti-miR-21-induced toxicity. Each experiment was performed in triplicate, ${ }^{* * *} \mathrm{P}<0.001,{ }^{* *} \mathrm{P}<0.01,{ }^{*} \mathrm{P}<0.05$.

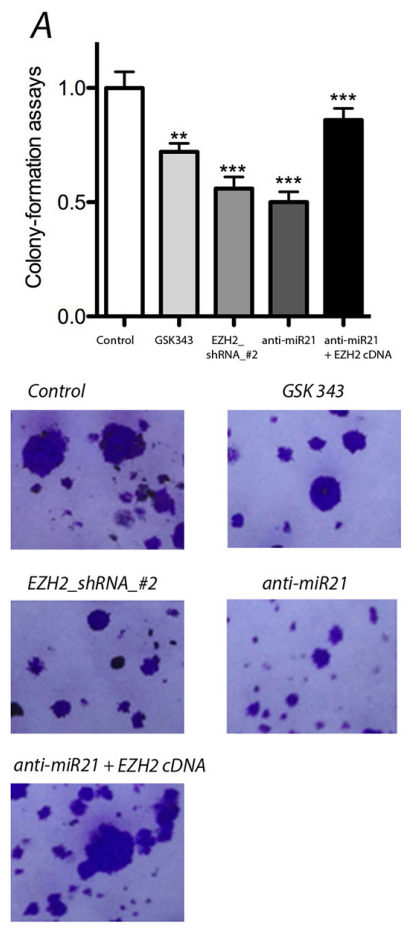

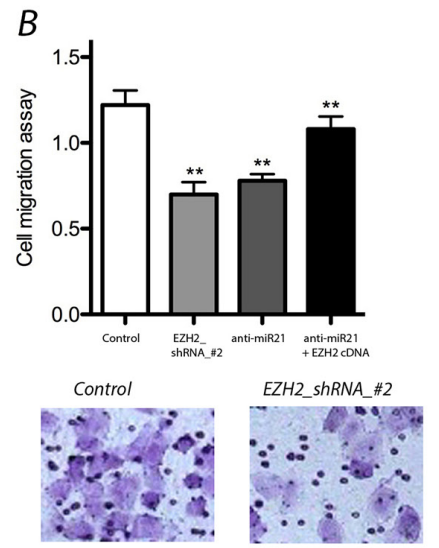
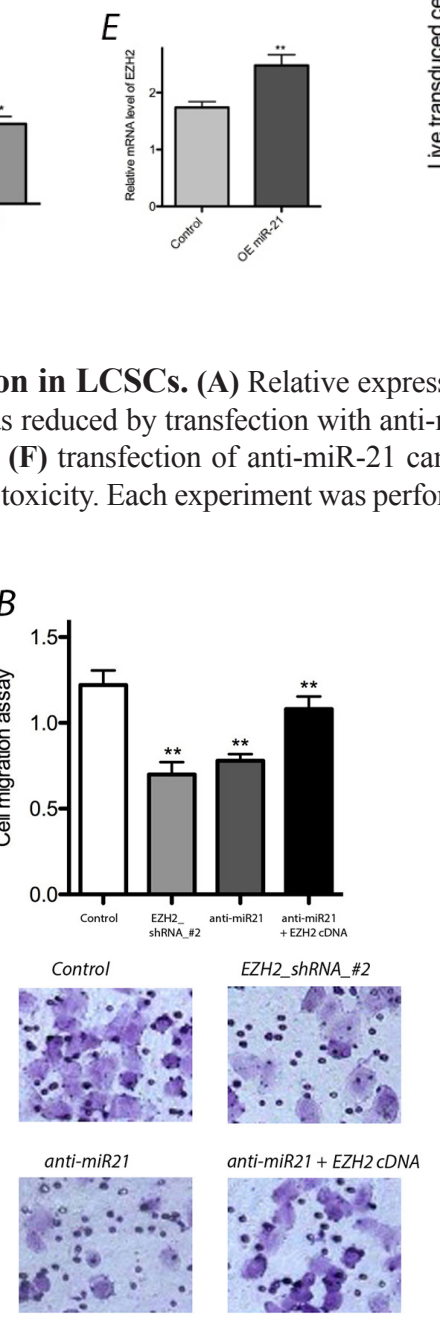
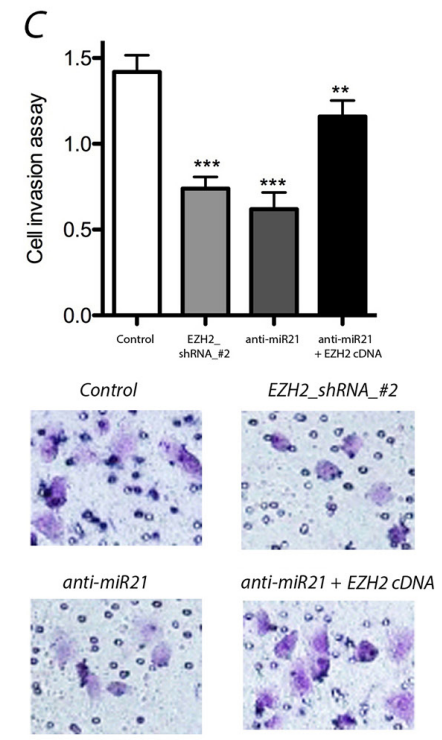

Figure 3: Effect of miR-21 and EZH2 on colony-formation, migration and invasion of LCSCs. (A) Colony-formation assays was decreased significantly after EZH2 or miR-21 knockdown, but rescued the effects of anti-miR-21 after overexpression of exogenous EZH2 in LCSCs; (B) \& (C) cell migration and invasion were reduced significantly by EZH2 or miR-21 knock down, but exogenous EZH2 over expression rescued the effects of anti-miR-21 in LCSCs. Each experiment was performed in triplicate, ${ }^{* * *} \mathrm{P}<0.001$, ${ }^{* *} \mathrm{P}<0.01,{ }^{*} \mathrm{P}<0.05$. 


\section{Effects on cell cycle}

We investigated the cell cycle alterations induced by the altered expression of miR-21 or EZH2 in LCSCs by FACS. We also evaluated the combinative effects of chemoor radiotherapy on cell cycle progression after altering the expression levels of miR-21 or EZH2 by transfection. Our results showed that the S-phase fraction of cells decreased by $13 \%$ or $22 \%$ in the presence of anti-miR-21 or EZH2 shRNA, respectively. Upon cisplatin treatment, the S-phase fraction of cells decreased by $11 \%$, but it decreased more significantly in the presence of anti-miR-21 or EZH2 shRNA (by $26 \%$ or $29 \%$, respectively). In addition, the number of cells in $\mathrm{G}_{2} / \mathrm{M}$ phase increased by $21 \%$ or $19 \%$ after transfection with anti-miR-21 or EZH2 shRNA, respectively, and it increased more significantly upon chemotherapeutic treatment by $32 \%$ or $35 \%$ in the presence of anti-miR21 or EZH2 shRNA, respectively (Supplementary Table 1).

Similar effects were observed upon radiotherapy, where additive changes were also observed. The S-phase fraction of cells decreased by $20 \%$ and $26 \%$ in the
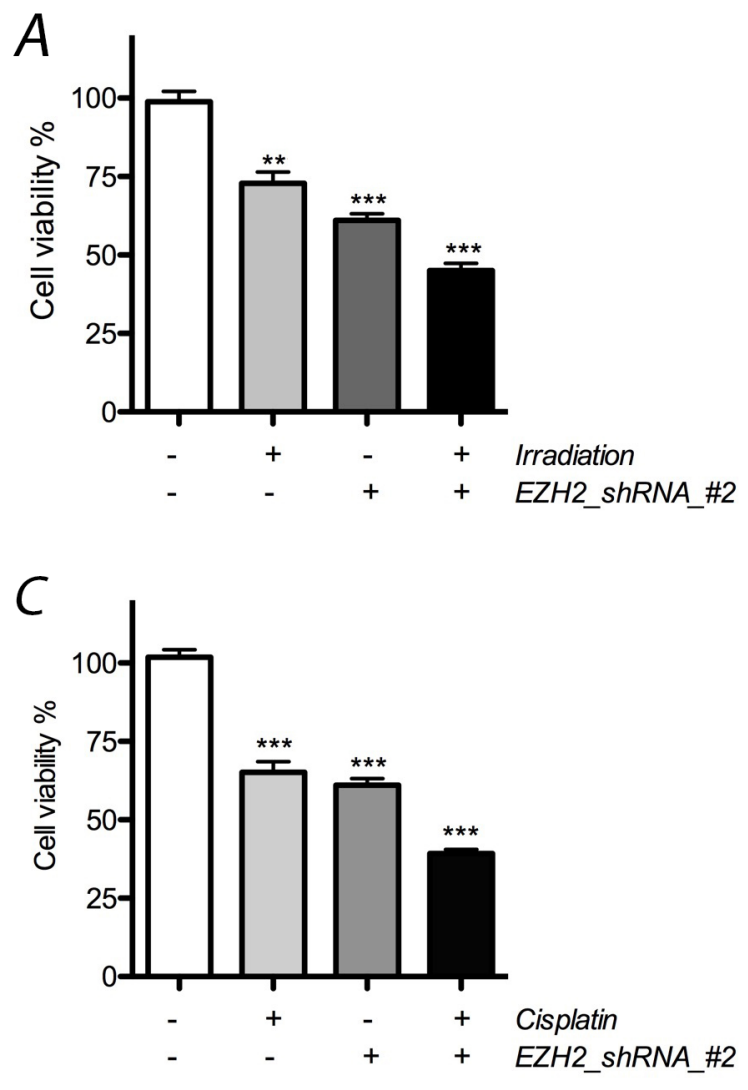

presence of anti-miR-21 or EZH2 shRNA, respectively, after combined treatment, whereas it only decreased by $13 \%$ after radiotherapy treatment. In contrast, the number of cells in $\mathrm{G}_{2} / \mathrm{M}$ phase increased by $41 \%$ or $38 \%$ after above combinatorial therapy but only resulted in a $23 \%$ increase after radiotherapy. Further experiments were also performed to observe the rescue effect of EZH2 up regulation (Supplementary Table 2).

We next detected the expression levels of cellcycle-related biomarkers including cell division cycle 2 (Cdc2) and cyclin B1 after transfection with anti-miR-21 or EZH2 shRNA by western blotting. Cdc2 and cyclin B1 decreased markedly both in presence of either antimiR-21 or EZH2 shRNA. After combined treatment with radio- or chemotherapy, $\mathrm{Cdc} 2$ and cyclin $\mathrm{B} 1$ also changed significantly (Figure 5, Supplementary Figure 2). Our results demonstrate that down regulation of $\mathrm{Cdc} 2$, cyclin B1 expression correlates with altered cell cycle dynamics after changes in miR-21 or EZH2 levels, which might help to improve the therapeutic effects of chemotherapy or radiation therapy on LCSCs.
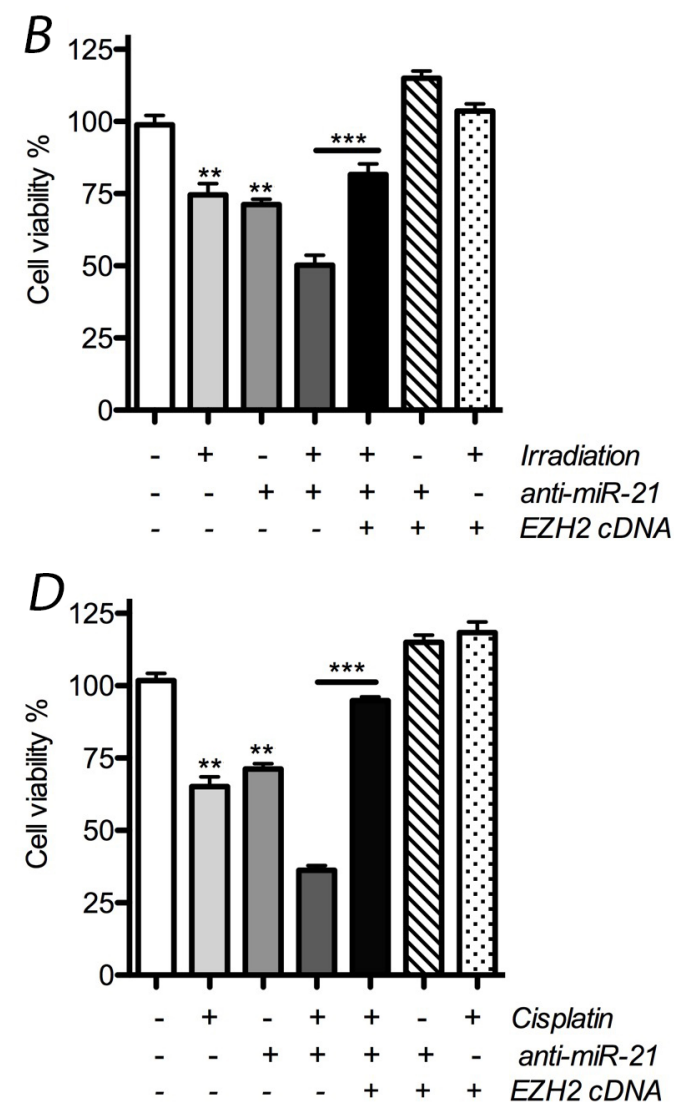

Figure 4: Effect of miR-21 or EZH2 on cell proliferation of LCSCs. (A) Combinative effect on cell proliferation was obtained obviously after treated with radiotherapy and EZH2_shRNA; (B) cell proliferation was reduced after combined with radiotherapy and antimiR-21, but rescued above effects after overexpression of exogenous EZH2 in LCSCs; (C) combinative effect on cell proliferation was obtained obviously after treated with cisplatin and EZH2_shRNA; (D) cell proliferation was reduced after combined with cisplatin and antimiR-21, but rescued above effects after ectopic expression of EZH2 in LCSCs. Each experiment was performed in triplicate, ${ }^{* * *} \mathrm{P}<0.001$, ${ }^{* * *} \mathrm{P}<0.01,{ }^{*} \mathrm{P}<0.05$. 


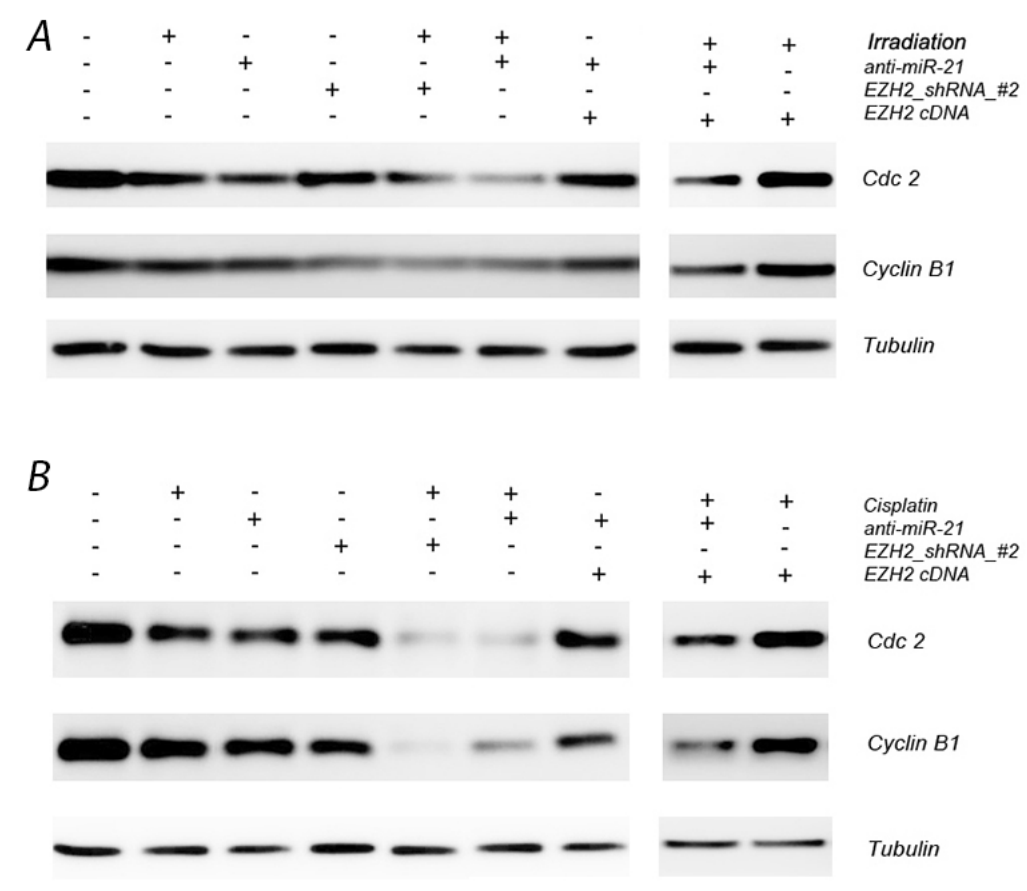

Figure 5: Effect of miR-21 or EZH2 on cell cell-cycle-related biomarkers in LCSCs. (A) Cdc2 and cyclin B1 expression after transfection with anti-miR-21 or EZH2 shRNA in the presence or absence of irradiation treatment on LCSCs; (B) Cdc2 and cyclin B1 expression after transfection with anti-miR-21 or EZH2 shRNA in the presence or absence of cisplatin treatment on LCSCs. Each experiment was performed in triplicate.
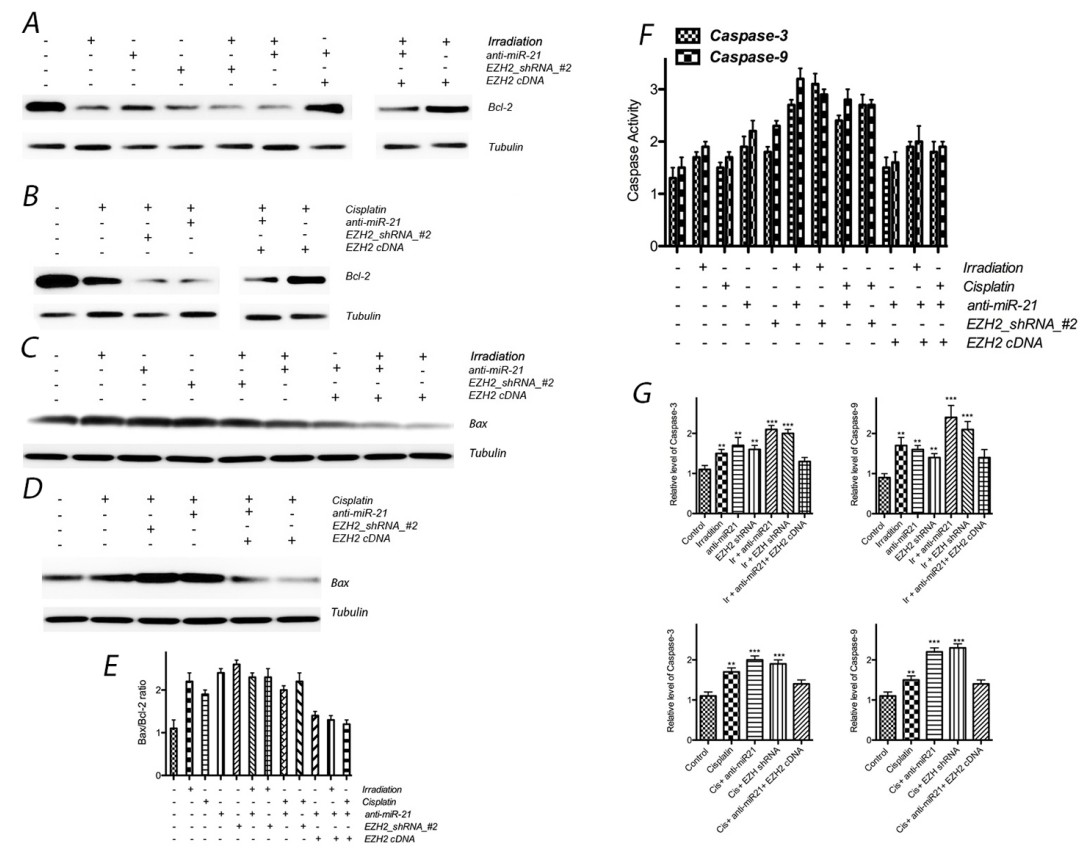

G

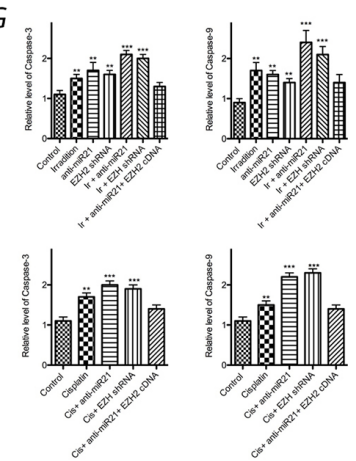

Figure 6: Effect of miR-21 or EZH2 on cell apoptosis of LCSCs. (A) Bcl-2 expression after transfection with anti-miR-21 or EZH2 shRNA in the presence or absence of irradiation treatment on LCSCs; (B) Bcl-2 expression after transfection with anti-miR-21 or EZH2 shRNA in the presence or absence of cisplatin treatment on LCSCs; (C) Bax expression after transfection with anti-miR-21 or EZH2 shRNA in the presence or absence of irradiation treatment on LCSCs; (D) Bax expression after transfection with anti-miR-21 or EZH2 shRNA in the presence or absence of cisplatin treatment on LCSCs; (E) the ratio between Bax and Bcl-2 also increased obviously after above treatment; (F) caspase activity were observed after above combinative treatment; (G) caspase exression were observed after above combinative treatment. Each experiment was performed in triplicate, ${ }^{* * *} \mathrm{P}<0.001,{ }^{* *} \mathrm{P}<0.01, * \mathrm{P}<0.05$. 


\section{Effects on apoptosis}

To evaluate the effects of miR-21 or EZH2 on LCSC apoptosis, we assessed apoptotic rates by FACS. The percentages of apoptotic cells were $11.2 \pm 0.3 \%$ and $12.1 \pm 1.1 \%$ in the presence of anti-miR-21 or EZH2 shRNA, respectively. In the single chemo- or radiation therapy groups, the percentages were $14.1 \pm 0.5 \%$ and 14.9 $\pm 0.2 \%$, respectively. In addition, additive effects on cell apoptosis were also observed after combined treatment, where the percentages were $18.5 \pm 0.3 \%$ and $21.9 \pm 0.9 \%$ after irradiation coupled with anti-miR-21 or EZH2 shRNA, respectively, and $21.3 \% \pm 0.2$ and $22.2 \pm 0.4 \%$ for chemotherapy coupled with anti-miR-21 or EZH2 shRNA, respectively (Supplementary Tables 3 and 4).

Furthermore, to assess the expression of apoptosisrelated molecular markers induced by the above treatments, we evaluated Bcl-2 and Bax expression by Western blotting. In addition, we assessed caspase- 3 and caspase-9 activities using an assay kit. After transfection of either anti-miR-21 or EZH2 siRNA, Bcl-2 expression markedly decreased and Bax expression increased, and thus the ratio between Bax and Bcl-2 also increased obviously after above treatment; caspase- 3 and caspase- 9 activities were increased significantly, in addition, expression of caspase- 3 and caspase- 9 were also enhanced after knock down miR-21 or EZH2. The expression of the above apoptosis-related molecular markers significantly decreased in the combined treatment group compared with those in the single-treatment groups. In addition, the exogenous expression of EZH2 also elicited a rescue effect against anti-miR21-induced apoptosis (Figure 6).

\section{DISCUSSION}

Our data provide the following novel findings: (1) increased expression of EZH2 and miR-21 is observed in LCSCs; (2) miR-21 overexpression increases EZH2 levels in LCSCs; (3) knock down of EZH2 and miR-21 sensitizes LCSCs to chemo- and radiation therapy; (4) exogenous expression of EZH2 rescues the effects of anti-miR-21; (5) the downstream effectors, including $\mathrm{Cdc} 2$, cyclin B1, Bax and $\mathrm{Bcl}-2$, which are involved in cell cycle progression and apoptosis, are up or down regulated by alterations in miR-21 and EZH2 levels in LCSCs. Thus, our data demonstrate the direct relationship between miR-21 and EZH2, which helps to explain the fact that these two molecules can enhance the biological behavior of LCSC through the modification of their targets. Such effects suggest the potential for targeting miR-21 and EZH2 to improve the therapeutic efficacy of lung cancer treatments.

Combining radio- or chemotherapy with biological agents for targeting cancer has been investigated to improve therapeutic outcomes and decrease toxicity to normal tissues due to specific tumor response, effects that were observed in our study. However, resistance to radioor chemotherapy, which impairs therapeutic efficacy, has been reported as significant reasons for the poor longterm survival for most NSCLS patients [39, 40]. Thus, investigations of new methods and therapeutic targeting, which can reduce resistance and increase therapeutic efficacy, have recently garnered increased attention. Our previous studies also demonstrated that the knock down EZH2 and miR-21 can sensitize radioresistant NSCLC A549 cells $[33,36]$. In the current study, the purpose was to test the hypothesis that the down regulation of EZH2 and miR-21 can have additive effects when combined with radiation or chemotherapy in LCSCs.

It has been reported that $\mathrm{EZH} 2$ is an oncogene involved in tumor progression, and miR-21 is also a biomarker for the tumorigenic processes, particularly for the pathological development of lung cancer [41, 42]. However, few studies have focused on these two molecules in LCSC. Our data provide positive evidence that EZH2 and miR-21 are highly expressed in LCSCs, which are suggestive of their important roles in the biological behavior of lung cancer.

In addition, few studies have focused on the up regulation of EZH2 and its underlying mechanisms. Thus, in this study, we experimentally evaluated the relationship between EZH2 and miR-21. Taken together, our results indicate that both miR-21 and EZH2 are essential for the proliferative potential and colony-formation capacity of lung cancer cells. The expression of EZH2, which can rescue the effects of anti-miR-21, could also be a down stream effector of miR-21.

EZH2 knockdown can increase the expression of p53 and p21, which can significantly modulate the levels of Cdc2 and cyclin B1 in both A549 and HTB-56 cells [36]. However, whether EZH2 can also elicit similar effects in LCSCs is unknown. Our results indicate that the down regulation of $\mathrm{EZH} 2$ or miR-21 can regulate cell cycle distribution, arresting cells in $\mathrm{G}_{2} / \mathrm{M}$ and delaying cell cycle progression. In addition, cell cycle dynamics were more significantly altered in the combination treatment groups compared with single treatment groups. The effects of miR-21 on cell cycle were rescued by the exogenous over expression of EZH2.

In conclusion, our study provides direct evidence that miR-21 and EZH2 knockdown can reduce the biological behavior of human lung cancer stem cells in vitro. Our data indicate that the altered behavior is associated with biomarkers of cell cycle or apoptosis. In addition, EZH2 might represent a down stream effector of miR-21. Our findings can potentially be used to develop new therapeutic targets and treatments for NSCLC.

\section{MATERIALS AND METHODS}

\section{Cell culture}

The human lung adenocarcinoma A549 cell line was obtained from the American Type Culture Collection (ATCC, Manassas, VA), SP cells (LCSCs) were prepared as 
previously reported [11-13] and maintained in Dulbecco's modified Eagle's medium (DMEM, Gibco, Carlsbad, $C A, U S A$ ) supplemented with $10 \%$ heat-inactivated FBS and $1 \%$ penicillin/ streptomycin at $37^{\circ} \mathrm{C}$ in a humidified incubator with $95 \%$ air and $5 \% \mathrm{CO}_{2}$. The serum free DMEM/F12 including EGF (20 ng/mL; Pepro Tech), bFGF (20 ng/mL; PeproTech) and IGF1 (20 ng/mL; PeproTech) was used for SP cell culture. All procedures had the approval of the Ethics Committee of the PLA General Hospital.

\section{Transfection}

Using Lipofectamine 2000 transfection reagent (Invitrogen, Carlsbad, CA, USA), cells were transfected with anti-miR-21 (5'-UCAACAUCAGUCUGA UAAGCUA-3') or the negative control (NC, 5 '-CAGUACUUUUG-UGUAGUACAA-3'), which were obtained from Ambion Inc. (Austin, TX, USA). In addition, miR-21 overexpression was achieved by transfecting cells with a synthetic miRIDIAN mimic or a negative control. Another group of cells was transfected with validated shRNA for EZH2 or a negative control vector (Qiagen, Lafayette, CO, USA) at a concentration of $100 \mathrm{nM}$ using the Lipofectamine 2000 transfection reagent. The EZH2 gene targeting sequences were as follows: EZH2 sense, TTCATGCAACACCCAACACT; and EZH2 antisense, GAGAGCAGCAGCAAACTCCT. Furthermore, EZH2mutant (NM_152998, Origene, Beijing, China) expression constructs were generated by cloning the corresponding cDNAs into the vector. At $6 \mathrm{~h}$ post-transfection, the medium was replaced with standard culture medium. After shRNA transfection, flow cytometry was performed to determine the toxicity of the shRNAs by quantifying the initial GFP-positive proportion of live cells. Doxycycline was used to induce the expression of the shRNA or exogenous cDNA expression.

\section{Western blotting}

Western blotting was performed as following protocol. Cells were lysed in buffer containing $0.01 \mathrm{M}$ Tris- $\mathrm{HCl}$ (pH 7.5), $150 \mathrm{mM} \mathrm{NaCl}, 0.01 \mathrm{M}$ EDTA, 0.01 M EGTA, 1\% Triton X-100, $0.01 \mathrm{M} \beta$-glycerophosphate, $0.01 \mathrm{M} \mathrm{Na}_{3} \mathrm{VO}_{4}$, and $1 \mu \mathrm{g} / \mathrm{ml}$ leupeptin supplemented with proteinase (Sigma-Aldrich, St. Louis, MO, USA) and phosphatase (Sigma-Aldrich, St. Louis, MO, USA) inhibitor cocktails. Protein concentrations of the cell lysates were determined using a BCA protein assay kit (Pierce, Rockford, IL, USA). All antibodies used in this study were obtained from Cell Signaling Technology (Beverly, MA, USA), and $\beta$-actin (Sigma-Aldrich, St. Louis, $M O, U S A$ ) was used to correct for potential unequal loading and transfer of proteins.

\section{Quantitative PCR}

The mRNA expression levels of miR-21 and EZH2 were determined by quantitative PCR using predesigned assays (Applied Biosystems, CA,USA).

\section{Colony-formation assay}

For the colony-formation assays, cells were seeded in triplicate into 6-well tissue culture plates and cultured for 12 days. Then, Giemsa staining was used to evaluate the number of colonies by light microscopy.

\section{Migration assay}

For the transwell migration assays, cells were seeded into the upper chamber in serum-free medium. The lower chamber contained growth medium with $10 \%$ $\mathrm{FBS}$ and $100 \mathrm{ng} / \mathrm{ml} \mathrm{EGF}$ (Invitrogen, USA). After $24 \mathrm{~h}$, the cells that had migrated to the lower chamber were fixed with $1 \%$ paraformaldehyde and stained with $1 \%$ crystal violet. Cell migration was quantified using light microscopy.

\section{Invasion assays}

Invasion was evaluated using the transwell system after transfection. Cells were seeded in the upper chamber that contained growth media with $10 \% \mathrm{FBS}$ and $100 \mathrm{ng} /$ $\mathrm{ml}$ EGF (Invitrogen, USA) for $24 \mathrm{~h}$. Next, cells that passed through the filter were fixed with $1 \%$ paraformaldehyde and stained with hematoxylin. The number of invaded cells was quantified using light microscopy.

\section{Radiation treatment and chemotherapy}

After the cells were transfected with anti-miR-21 or EZH2 shRNA, the cells in the radiation-treated group were $\gamma$-irradiated at a single dose of $2 \mathrm{~Gy} / \mathrm{min}$ every 3 days for 2 weeks, and the cells in the chemotherapy group were treated with $30 \mathrm{nM}$ cisplatin every 3 days for 2 weeks.

\section{Cell proliferation}

Cell proliferation was evaluated using an MTT kit (Roche Diagnostics, Germany) after radio- or chemotherapy in the presence or absence of the transfections described above. Cells were seeded at a density of $5 \times 10^{3}$ cells/well and were treated as described above. The cells in each treatment group were harvested by trypsinization at different time points, and the results were recorded using a Universal Microplate Spectrophotometer (BioTek, VT, USA). Experiments were performed in triplicate. 


\section{Cell cycle analysis}

To evaluate the cell cycle phase distribution, cells were seeded at a density of $1 \times 10^{5}$ cells/well in six-well plates. As described in the protocol above, the cells were transfected and treated with radio- or chemotherapy. Next, the cells were harvested, washed and fixed with $70 \%(\mathrm{v} / \mathrm{v})$ ethanol overnight at $4{ }^{\circ} \mathrm{C}$. RNase A $(100 \mu \mathrm{g} /$ $\mathrm{ml}$ ) and propidium iodide (PI, $50 \mathrm{mg} / \mathrm{ml}$ ) were incubated with cells for $30 \mathrm{~min}$. The cell cycle phase distribution was assessed by flow cytometry (Becton Dickinson, San Jose, CA, USA). The experiments were performed in triplicate. Primary antibodies against $\mathrm{Cdc} 2$ and cyclin B1 (1:1,000; Cell Signaling Technology, Beverly, MA, USA) were used to assess protein expression by western blotting.

\section{Cell apoptosis analysis}

To analyze apoptosis, cells were seeded at a density of $1 \times 10^{5}$ cells/well in six-well plates. As detailed above, the cells were transfected and treated with radioor chemotherapy. The cells were incubated with PI and annexin V-FITC to assess apoptosis by flow cytometry (Becton Dickinson, San Jose, CA, USA). The primary antibody against Bax and Bcl-2 (1:1000; Santa Cruz Biotechnology, CA, USA) was used to assess its expression by western blotting. Caspase activities was also observed by the specific assay kit.

\section{Author contributions}

H.X., and Y.L. designed the plan. H.X., W.Z., B. Z., and Y.Z. performed the experiments. H.X., Y.Z. and S.L. analysed the results. H.X., and Y.L. wrote the manuscript. All authors reviewed the manuscript.

\section{CONFLICTS OF INTEREST}

The authors declare that they have no competing interests. All authors reviewed and approved the final version of the manuscript.

\section{REFERENCES}

1. Fidias P, Novello S. Strategies for prolonged therapy in patients with advanced non-small-cell lung cancer. J Clin Oncol. 2010;28:5116-23.

2. Fuld AD, Dragnev KH, Rigas JR. Pemetrexed in advanced non-small-cell lung cancer. Expert Opin Pharmacother. 2010;11:1387-402.

3. Whitehurst AW, Bodemann BO, Cardenas J, Ferguson D, Girard L, Peyton M, Minna JD, Michnoff C, Hao W, Roth MG, Xie XJ, White MA. Synthetic lethal screen identification of chemosensitizer loci in cancer cells. Nature. 2007;446:815-9.
4. Schild SE, Vokes EE. Pathways to improving combined modality therapy for stage III nonsmall-cell lung cancer. Ann Oncol. 2016;27:590-9.

5. Fennell DA, Summers Y, Cadranel J, Benepal T, Christoph DC, Lal R, Das M, Maxwell F, Visseren-Grul C, Ferry D. Cisplatin in the modern era: the backbone of first-line chemotherapy for non-small cell lung cancer. Cancer Treat Rev. 2016;44:42-50.

6. Rooney C, Sethi T. Advances in molecular biology of lung disease: aiming for precision therapy in non-small cell lung cancer. Chest. 2015;148:1063-72.

7. Bozorgi A, Khazaei M, Khazaei MR. New findings on breast cancer stem cells: a review. J Breast Cancer. 2015;18:303-12.

8. Sharma N, Nanta R, Sharma J, Gunewardena S, Singh KP, Shankar S, Srivastava RK. PI3K/AKT/mTOR and sonic hedgehog pathways cooperate together to inhibit human pancreatic cancer stem cell characteristics and tumor growth. Oncotarget. 2015;6:32039-60. https://doi. org/10.18632/oncotarget.5055.

9. Seymour T, Nowak A, Kakulas F. Targeting aggressive cancer stem cells in glioblastoma. Front Oncol. 2015;5:159.

10. Leon G, MacDonagh L, Finn SP, Cuffe S, Barr MP. Cancer stem cells in drug resistant lung cancer: targeting cell surface markers and signaling pathways. Pharmacol Ther. 2016;158:71-90.

11. Bertolini G, Roz L, Perego P, Tortoreto M, Fontanella E, Gatti L, Pratesi G, Fabbri A, Andriani F, Tinelli S, Roz E, Caserini R, Lo Vullo S, et al. Highly tumorigenic lung cancer CD133+ cells display stem-like features and are spared by cisplatin treatment. Proc Natl Acad Sci U S A. 2009;106:16281-6.

12. Suresh R, Ali S, Ahmad A, Philip PA, Sarkar FH. The role of cancer stem cells in recurrent and drug-resistant lung cancer. Adv Exp Med Biol. 2016;890:57-74.

13. Xia H, Yu C, Zhang W, Zhang B, Zhao Y, Fang F. Identification and isolation of cancer stem cells from A549 cells. Chin J Lung Cancer. 2013;16:400-4.

14. Stella GM, Luisetti M, Pozzi E, Comoglio PM. Oncogenes in non-small-cell lung cancer: emerging connections and novel therapeutic dynamics. Lancet Respir Med. 2013;1:251-61.

15. Capelletto E, Novello S. Emerging new agents for the management of patients with non-small cell lung cancer. Drugs. 2012;72:37-52.

16. Kuykendall A, Chiappori A. Advanced EGFR mutationpositive non-small-cell lung cancer: case report, literature review, and treatment recommendations. Cancer Control. 2014;21:67-73.

17. Behrens C, Solis LM, Lin H, Yuan P, Tang X, Kadara H, Riquelme E, Galindo H, Moran CA, Kalhor N, Swisher SG, Simon GR, Stewart DJ, et al. EZH2 protein expression associates with the early pathogenesis, tumor progression, and prognosis of non-small cell lung carcinoma. Clin Cancer Res. 2013;19:6556-65. 
18. Gonzalez ME, DuPrie ML, Krueger H, Merajver SD, Ventura AC, Toy KA, Kleer CG. Histone methyltransferase EZH2 induces Akt-dependent genomic instability and BRCA1 inhibition in breast cancer. Cancer Res. 2011;71:2360-70.

19. Gonzalez ME, Li X, Toy K, DuPrie M, Ventura AC, Banerjee $\mathrm{M}$, Ljungman $\mathrm{M}$, Merajver SD, Kleer CG. Downregulation of EZH2 decreases growth of estrogen receptor-negative invasive breast carcinoma and requires BRCA1. Oncogene. 2009;28:843-53.

20. Moore HM, Gonzalez ME, Toy KA, Cimino-Mathews A, Argani P, Kleer CG. EZH2 inhibition decreases p38 signaling and suppresses breast cancer motility and metastasis. Breast Cancer Res Treat. 2013;138:741-52.

21. Verma SK. Inhibition of the histone lysine methyltransferase EZH2 for the treatment of cancer. Curr Top Med Chem. 2015;15:714-9.

22. Tong ZT, Cai MY, Wang XG, Kong LL, Mai SJ, Liu YH, Zhang HB, Liao YJ, Zheng F, Zhu W, Liu TH, Bian XW, Guan XY, et al. EZH2 supports nasopharyngeal carcinoma cell aggressiveness by forming a co-repressor complex with HDAC1/HDAC2 and Snail to inhibit E-cadherin. Oncogene. 2012;31:583-94.

23. Fan $\mathrm{T}$, Jiang S, Chung $\mathrm{N}$, Alikhan A, Ni C, Lee CC, Hornyak TJ. EZH2-dependent suppression of a cellular senescence phenotype in melanoma cells by inhibition of p21/CDKN1A expression. Mol Cancer Res. 2011;9:418-29.

24. Inamura K, Ishikawa Y. MicroRNA in lung cancer: novel biomarkers and potential tools for treatment. J Clin Med. 2016;5.

25. Naidu S, Magee P, Garofalo M. MiRNA-based therapeutic intervention of cancer. J Hematol Oncol. 2015;8:68.

26. Nadal E, Truini A, Nakata A, Lin J, Reddy RM, Chang AC, Ramnath N, Gotoh N, Beer DG, Chen G. A novel serum 4-microRNA signature for lung cancer detection. Sci Rep. 2015;5:12464.

27. Capodanno A, Boldrini L, Proietti A, Alì G, Pelliccioni S, Niccoli C, D'Incecco A, Cappuzzo F, Chella A, Lucchi M, Mussi A, Fontanini G. Let-7g and miR-21 expression in non-small cell lung cancer: correlation with clinicopathological and molecular features. Int J Oncol. 2013;43:765-74.

28. Singh M, Garg N, Venugopal C, Hallett R, Tokar T, McFarlane N, Mahendram S, Bakhshinyan D, Manoranjan B, Vora P, Qazi M, Arpin CC, Page B, et al. STAT3 pathway regulates lung-derived brain metastasis initiating cell capacity through miR-21 activation. Oncotarget. 2015;6:27461-77. https://doi.org/10.18632/oncotarget.4742.

29. Frezzetti D, De Menna M, Zoppoli P, Guerra C, Ferraro A, Bello AM, De Luca P, Calabrese C, Fusco A, Ceccarelli M, Zollo M, Barbacid M, Di Lauro R, et al. Upregulation of miR-21 by Ras in vivo and its role in tumor growth. Oncogene. 2011;30:275-86.

30. Misawa A, Katayama R, Koike S, Tomida A, Watanabe T, Fujita N. AP-1-Dependent miR-21 expression contributes to chemoresistance in cancer stem cell-like SP cells. Oncol Res. 2010;19:23-33.
31. Giovannetti E, Funel N, Peters GJ, Del Chiaro M, Erozenci LA, Vasile E, Leon LG, Pollina LE, Groen A, Falcone A, Danesi R, Campani D, Verheul HM, et al. MicroRNA-21 in pancreatic cancer: correlation with clinical outcome and pharmacologic aspects underlying its role in the modulation of gemcitabine activity. Cancer Res. 2010;70:4528-38.

32. Yan M, Chen C, Gong W, Yin Z, Zhou L, Chaugai S, Wang DW. miR-21-3p regulates cardiac hypertrophic response by targeting histone deacetylase- 8 . Cardiovasc Res. 2015;105:340-52.

33. Ma Y, Xia H, Liu Y, Li M. Silencing miR-21 sensitizes non-small cell lung cancer A549 cells to ionizing radiation through inhibition of PI3K/Akt. Biomed Res Int. 2014;2014:617868.

34. McCabe MT, Graves AP, Ganji G, Diaz E, Halsey WS, Jiang Y, Smitheman KN, Ott HM, Pappalardi MB, Allen KE, Chen SB, Della Pietra A 3rd, Dul E, et al. Mutation of A677 in histone methyltransferase EZH2 in human B-cell lymphoma promotes hypertrimethylation of histone H3 on lysine 27 (H3K27). Proc Natl Acad Sci U S A. 2012;109:2989-94.

35. Vella S, Pomella S, Leoncini PP, Colletti M, Conti B, Marquez VE, Strillacci A, Roma J, Gallego S, Milano GM, Capogrossi MC, Bertaina A, Ciarapica $\mathrm{R}$, et al. MicroRNA-101 is repressed by EZH2 and its restoration inhibits tumorigenic features in embryonal rhabdomyosarcoma. Clin Epigenetics. 2015;7:82.

36. Xia H, Zhang W, Li Y, Guo N, Yu C. EZH2 silencing with RNA interference induces $\mathrm{G} 2 / \mathrm{M}$ arrest in human lung cancer cells in vitro. Biomed Res Int. 2014;2014:348728.

37. Bondgaard AL, Poulsen TT, Poulsen HS, Skov BG. Different expression of EZH2, BMI1 and Ki67 in low and high grade neuroendocrine tumors of the lung. Cancer Biomark. 2012;11:123-8.

38. Hayashi S, Kumai T, Matsuda Y, Aoki N, Sato K, Kimura S, Kitada M, Tateno M, Celis E, Kobayashi H. Sixtransmembrane epithelial antigen of the prostate and enhancer of zeste homolog 2 as immunotherapeutic targets for lung cancer. J Transl Med. 2011;9:191.

39. Simone CB, Burri SH, Heinzerling JH. Novel radiotherapy approaches for lung cancer: combining radiation therapy with targeted and immunotherapies. Transl Lung Cancer Res. 2015;4:545-52.

40. Santarpia M, Daffinà MG, Karachaliou N, González-Cao M, Lazzari C, Altavilla G, Rosell R. Targeted drugs in smallcell lung cancer. Transl Lung Cancer Res. 2016;5:51-70.

41. Fillmore CM, Xu C, Desai PT, Berry JM, Rowbotham SP, Lin YJ, Zhang H, Marquez VE, Hammerman PS, Wong KK, Kim CF. EZH2 inhibition sensitizes BRG1 and EGFR mutant lung tumours to TopoII inhibitors. Nature. 2015;520:239-42.

42. Markou A, Sourvinou I, Vorkas PA, Yousef GM, Lianidou E. Clinical evaluation of microRNA expression profiling in non small cell lung cancer. Lung Cancer. 2013;81:388-96. 